\title{
Preconditioned Reflection Full Waveform Inversion for Subsalt Modeling Building
}

\author{
Chao Wang ${ }^{*}$, Paul Farmer, David Yingst, John Brittan, Ian Jones, and Carlos Calderon, ION
}

Copyright 2019, SBGf - Sociedade Brasileira de Geofísica

This paper was prepared for presentation during the $16^{\text {th }}$ International Congress of the Brazilian Geophysical Society held in Rio de Janeiro, Brazil, 19-22 August 2019.

Contents of this paper were reviewed by the Technical Committee of the $16^{\text {th }}$ International Congress of the Brazilian Geophysical Society and do not necessarily represent any position of the SBGf, its officers or members. Electronic reproduction or storage of any part of this paper for commercial purposes without the written consent of the Brazilian Geophysical Society is prohibited.

\section{Abstract}

Reflection full waveform inversion (RFWI) has become an effective tool to generate a low-wavenumber deep model update using reflection energy and to improve seismic imaging, especially in the subsalt area. However it is still a challenging inverse problem that suffers from local minima issues with conventional data fitting objective function. To tackle this problem, we propose a novel weighted traveltime based objective function that focuses on correcting the kinematic errors between the field and synthetic data and derive a new calculation of the gradient from forming an adjoint source that is less likely to cycle-skip, instead of simply minimizing the residual for data fitting objective function. We also proposed a preconditioned optimization scheme to mitigate the artifacts and improve convergence rate. The potential benefit of our proposed preconditioned RFWI is to generate a deeper reliable low-wavenumber update with reflected energy in absence of a good starting background model and ultra low frequencies. The inverted model from this approach will provide a better initial model for conventional FWI and/or RFWI with data fitting objective functions. The proposed workflow will eventually lead to high-fidelity earth model for subsalt imaging and interpretation. Our kinematic based objective function and optimization scheme are not limited to RFWI, it can be also applied to FWI and forms preconditioned FWI, depending on the type of wave propagation and the component of the model we are trying to invert for.

\section{Introduction}

Conventional full waveform inversion (FWI) has been an essential tool to generate high-fidelity earth models for better seismic imaging and structural interpretation. It solves a least squares problem by minimizing the misfit between the acquired and synthetic data (Lailly, 1983; Tarantola, 1984; Virieux and Operto, 2009). Considering the intrinsic problem for conventional FWI, the success in providing reliable background models heavily relies on the offset range and low frequency content in the acquired data. With limited offset range and low frequency data, reliable background updates are usually constrained by the depth penetration from refraction energy with only shallow improvements to the model. We have to make use of reflection data in order to obtain a deeper update for a smooth background model. However using reflection data with conventional FWI while missing lowwavenumbers, only the high-wavenumbers of the target model can be inverted due to the dominant contribution from the migration kernel on the tomographic gradient. A different objective function that can separate the tomographic from the migration contribution is required for a successful RFWI. Various approaches have been proposed to benefit from the reflection energy. Van Leeuwen and Hermann (2013) introduced a penalized objective function for FWI with a reconstructed wavefield method. By reconstructing an extended source to generate reflections, the computed gradient with respect to velocity model results in a deeper low-wavenumber update with proper choice of penalty parameter (Wang et al., 2016). Alternatively, we can also use a density or reflectivity model to generate reflections, and then formulate an objective function as a function of the smooth background model (Xu et al., 2012). In this paper, the Born approximation is used to generate reflection data while both the velocity model and the reflectivity model are updated for each iteration.

Inverting reflections with $\mathrm{FWI}$ is a highly nonlinear problem and convergence to local minima is a challenge. A limitation for inverting reflections then becomes the need for good starting models. Over the last decade, various efforts have been made to mitigate the problems of local minima and many alternative methods have been proposed (Shen and Symes, 2008; Van Leeuwen and Hermann, 2013; Biondi and Almomin, 2014; Warner and Guasch, 2016; Wang et al., 2016; Huang et al., 2016; Vigh et al., 2016; Luo et al., 2016). All these previous works and our proposed method in this paper aim to avoid convergence to local minima by adding additional parameters to the model and expanding the search space in the hope that eventually the non-physical model converges to a physical one. By adopting an objective function that automatically adjusts for the poorly matched events, our approach is less likely to cycle-skip and in the meanwhile provides a deeper background model update by using reflected energy. We propose a preconditioning optimization algorithm to suppress artifacts introduced by conventional RFWI and thereby increase the convergence rate.

This paper first presents the theory and methodology for our 3D time domain preconditioned RFWI. It compares our proposed method with a conventional least squares objective function to demonstrate the benefits of our new objective function and optimization algorithm using 2D synthetic examples. Finally, a 3D field example will be shown to demonstrate the production applicability for subsalt velocity modeling building and imaging. 


\section{Theory and Method}

We first split the velocity model into a long wavelength component $\mathrm{m}$ and a short wavelength component $\mathrm{r}$ and apply the first order Born approximation to the acoustic wave equation. Then we have the following duopropagator for Born modeling (Hudson and Heritage, 1980; Aki and Richards, 1980)

$$
\begin{aligned}
& F[m] u=f, \\
& F[m] u=r{ }_{t}^{2} u .
\end{aligned}
$$

where $\mathrm{m}$ represents the subsurface smooth background velocity model, $r$ represents the reflectivity model, $F[\mathrm{~m}]$ is the wave operator or D'Alembert operator, $u$ is the forward propagated transmitted wavefield and $u$ is the forward propagated reflected wavefield, $f$ is the source function. Let S[m] denote the solution operator for the above linearized forward propagated wave equations. Then we can compute the forward transmitted wavefield

$$
u=S[m] f,
$$

and the forward reflected wavefield

$$
u=S[m] r{ }_{t}^{2} S[m] f .
$$

Given an initial background velocity model $\mathrm{m}$ and $\mathrm{a}$ reflectivity model $r$, our proposed objective function optimizes over smooth background model $\mathrm{m}$ to minimize the local travel time error between the synthetic data and the field data. It emphasizes the kinematics and is implemented in the time domain using high-order finite difference scheme. The forward modeled data are obtained from solving the wave equations with a first order Born approximation and time shift traces are computed from localized cross-correlation between the acquired field data and the predicted synthetic data. Luo and Schuster (1991) introduced the travel-time based objective function. We modified it so that our time error becomes a local measurement, which is a function of time and space. Our modified version can be beneficial when a single time-shift per trace is not adequate for handling complicated scenarios. Our proposed optimization problem is

$$
\begin{array}{ll}
\min _{m} & J[m]=\frac{1}{2}\left\|A_{0}\right\|_{2}^{2}, \\
\text { s.t. } & { }_{0}\left(x_{r}, t ; x_{s}\right)=\operatorname{argmax} \quad c\left(, x_{r}, t ; x_{s}\right),
\end{array}
$$

where $\mathrm{c}$ the windowed cross-correlation function at each trace sample, A is the weighting operator, is a time lag and ${ }_{0}$ is the optimal time lag function of time, receiver, and source, computed from maximizing the windowed cross-correlation. From implicit differentiation and the midpoint rule, we can estimate the new approximate adjoint source $R$. The gradient for computing descent direction can then be expressed as

$$
\nabla_{m} J=\frac{2}{m^{3}}\left\langle\partial_{t}^{2} u, S^{*} P^{*} R\right\rangle+\frac{2}{m^{3}}\left\langle\partial_{t}^{2} u, S^{*} r \partial_{t}^{2} S^{*} P^{*} R\right\rangle,
$$

where $\mathrm{P}$ is the restriction operator (a projection) that records the forward modeled wavefield at the receiver locations. As we noticed, the tomographic kernel from RFWI may introduce strong artifacts from the "rabbit ears" and also a sharp velocity contrast does not satisfy the Born assumptions. We design a preconditioner $\mathrm{C}$ by the change of variables $v=\mathrm{Cm}$, then the conjugate gradient (CG) descent direction for preconditioned model $v$ at $k+1^{\text {th }}$ iteration can be modified as follows

$$
\begin{aligned}
v_{k+1} & =v_{k}+{ }_{k} d_{k} \\
d_{k+1} & =C C^{T} g_{k+1}+{ }_{k} d_{k}
\end{aligned}
$$

Where ${ }_{k}$ is the steplength, and $g_{k}$ is the gradient, $d_{k}$ is the steepest descent direction. The preconditioned CG improves the convergence rate for RFWI with less artifacts and better updates. Since our preconditioned RFWI utilizes a kinematic based objective function, which quantifies local move out errors between reflection events, it reduces the risk of converging to local minima with an inaccurate initial model, compared with those using the conventional data misfit norm, and provides a deeper reliable background update with reflected energy and higher convergence rate. This approach is not limited to RFWI, it is also an effective method for mitigating the problem with cycle-skipping and increasing convergence rates for conventional FWI. Similarly we may replace the data residual for least squares FWI with the same adjoint source as we used here for formulating the corresponding gradient.

\section{Synthetic Examples}

We now demonstrate our method on a 2D synthetic example. Field data were generated with a Born finite difference method with a single reflector at $5000 \mathrm{~m}$ depth and constant background velocity of $2000 \mathrm{~m} / \mathrm{s}$ with a single source receiver pair. Our first initial model had an incorrect constant velocity $2200 \mathrm{~m} / \mathrm{s}$. In contrast to descent direction of least squares (LS) RFWI in Figure 1 (a), the preconditioned RFWI in Figure 1(b) produces the correct direction for updating the model. Figures 1(c) and 1 (d) show the case where the starting model is slower by $10 \%$ than the true model. Comparing with the descent direction for least squares RFWI shown in Figure 1(c), the preconditioned RFWI in Figure 1(d) provides us with a much better descent direction that will lead to faster convergence.

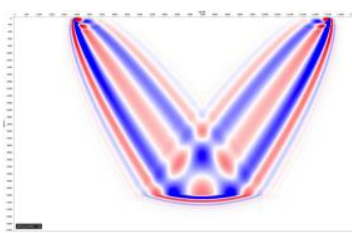

(a) LS with fast velocity

(a) LS with slow velocity

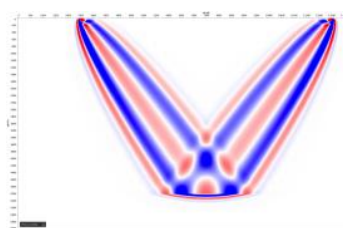

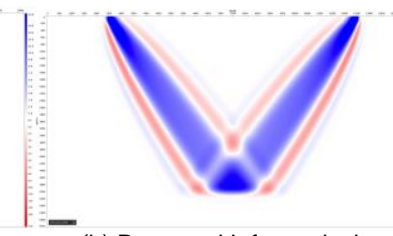

(b) Precon with fast velocity

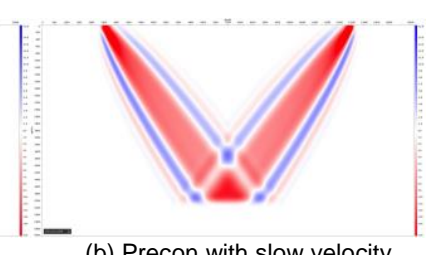

(b) Precon with slow velocity
Figure 1: Rabbit ear example 
Next we demonstrate the advantage of the proposed approach in the Marmousi dataset. The initial model shown in Figure 2(a) is a simple gradient model. The true model is shown in Figure 2(b), and was used to generate the field data set comprised of 59 common shot gathers with a shot spacing of $200 \mathrm{~m}$. Each shot gather contains 241 receivers with an interval of $20 \mathrm{~m}$. The lowest frequency used for inversion was $3 \mathrm{~Hz}$ and the maximum offset was $4800 \mathrm{~m}$. Figure 2(c) shows the inversion result from least squares (LS) conventional FWI while missing a good starting model and low frequency data. Although the model captures some of the true velocity trend, it converged to a local minimum with incorrect layer velocities. Our preconditioned RFWI generated reasonable updates however it has not converged to the true model. In contrast it produced a less detailed background model that can be used as a more accurate starting model for conventional FWI. Using inverted result from preconditioned RFWI as a starting model, conventional $\mathrm{FWI}$ converged to a more correct model shown in Figure 2(e).

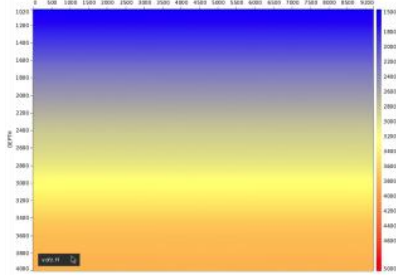

(a) Initial velocity

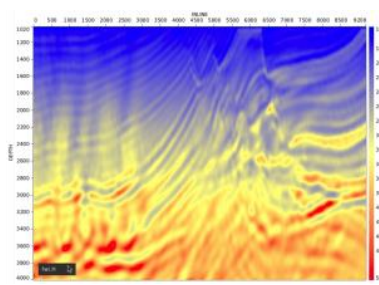

(c) LS

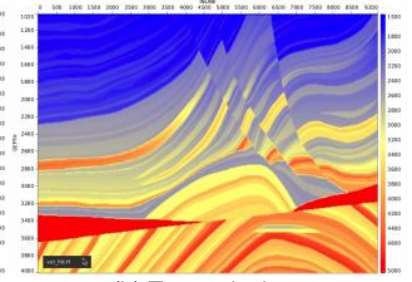

(b) True velocity

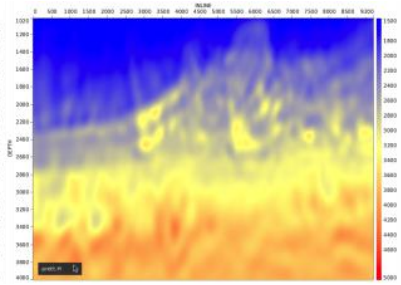

(b) Precon

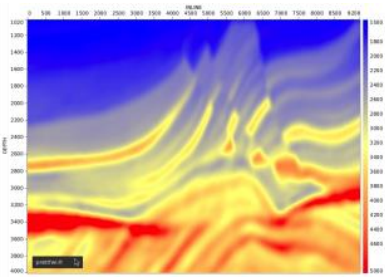

(b) Precon + LS

Figure 2: Marmousi example

\section{Results}

We finally present another application to a 3D streamer data set. This narrow azimuth seismic survey was located in the Campeche area offshore Mexico. The maximum offset was $6200 \mathrm{~m}$ and the lowest frequency used for inversion was $3 \mathrm{~Hz}$. Figure 3(a) shows the initial velocity that was used for inversion with a maximum depth of $10000 \mathrm{~m}$ overlaying the initial stack image and Figure 3(b) shows the inverted model from preconditioned reflection
FWI with the updated stack image. The image using the inverted model in Figure 3(b) shows improvements at the base salt and subsalt region indicated in the highlighted areas of the images with respect to Figure 3(a). Comparing the modeled shot gathers overlaying the field shot gathers with initial model in Figure 4(a), our method automatically aligned the events to avoid cycle-skipping with an improved fit around the first arrivals shown in Figure 4(b) and 4(c). The jitter in the shifted final modeled data (Figure $4(\mathrm{c})$ ) is caused by areas of low $\mathrm{S} / \mathrm{N}$ in the field data and will have little influence on the final result due to the application of the preconditioner. Preconditioned RFWI can provide a better reference model for FWI and a deeper update such as in subsalt GOM scenarios.

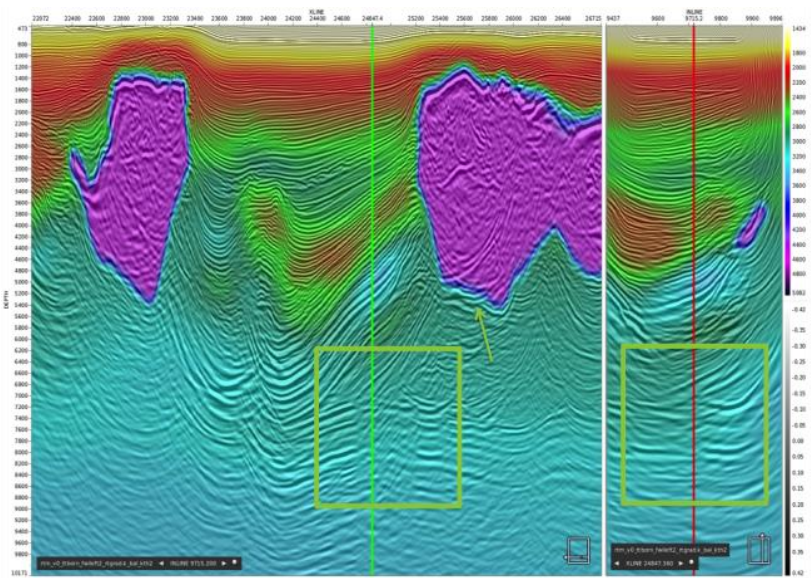

(a) Initial velocity with initial stack

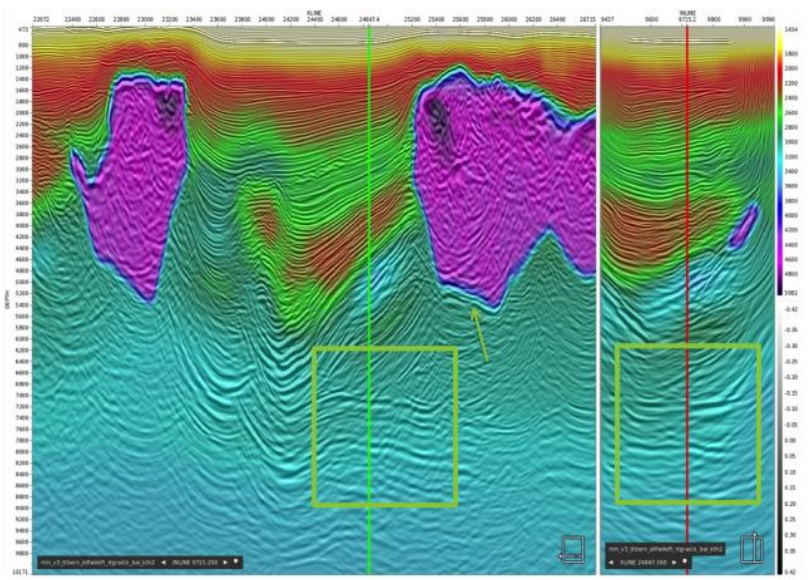

(b) Inverted velocity with updated stack

Figure 3: GOM example

\section{Conclusions}

We presented the theory and applications of our proposed inversion method, preconditioned reflection FWI. We formulated a new objective function that is based on the weighted traveltime shifts with the correction of the kinematics errors. Therefore it helps avoid cycle skipping issues to overcome some of the problems with 
local minima and relaxes the requirement for successful inversion. Additionally, we proposed a preconditioned optimization scheme to mitigate the artifacts and improve convergence rate. The results showed successful low wavenumber update from reflections with improved subsalt images.

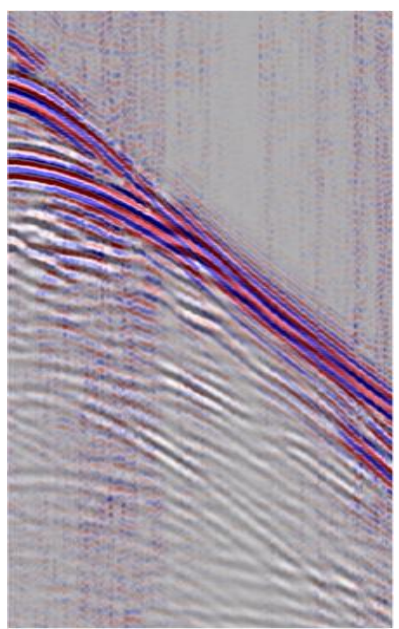

(a) Initial synthetic

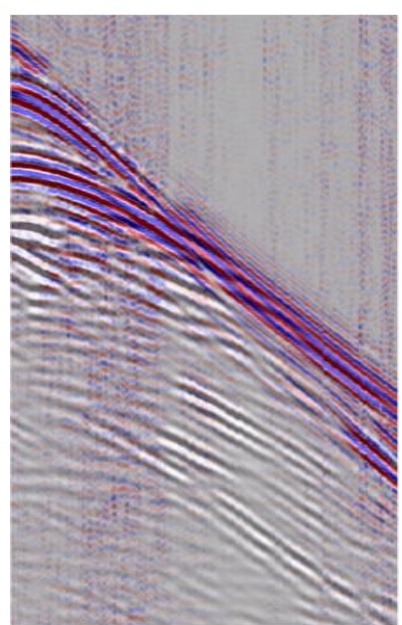

(b) Updated synthetic

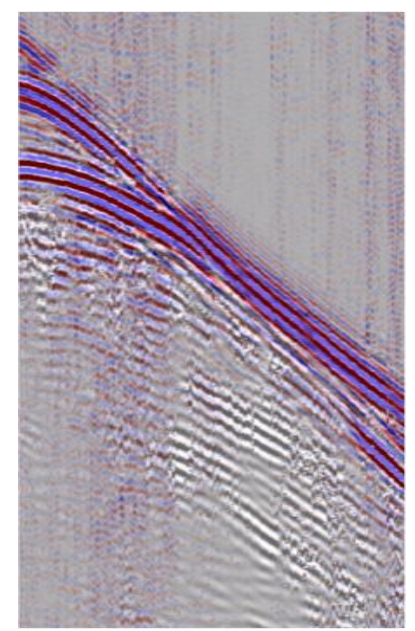

(c) Updated synthetic with kinematic correction

Figure 4: Shot gathers

\section{Acknowledgments}

We would like to thank ION for permission to publish the results. The Campeche data was reprocessed and reimaged by ION in partnership with Schlumberger, who holds data licensing rights. We also thank our colleagues for valuable supports.

\section{References}

Aki, K. and Richards, P. [1980] Quantitative Seismology, Theory and Methods.
Biondi, B. and Almomin, A. [2014] Simultaneous inversion of full data bandwidth by tomographic full waveform inversion. Geophysics, 79(3), WA129-WA140.

Huang, H., Symes,W. and Nammour, R. [2016] Matched source waveform inversion: Volume extension. Expanded Abstracts, Society of Exploration Geophysicists, Dallas, TX, 1364-1368.

Hudson, J. and Heritage, J. [1980] The use of the Born approximation in seismic scattering problems. Geophysical Journal of the Royal Astronomical Society, $66,221-240$.

Lailly, P. [1983] The Seismic Inverse Problem as a Sequence of Before-Stack Migrations. In: Bednar, J. (Ed.) Conference on Inverse Scattering: Theory and Applications, Society for Industrial and Applied Mathematics, Philadelphia, 206-220.

Luo, Y., Ma, Y., Wu, Y., Liu, H. and Cao, L. [2016] Fulltraveltime inversion. Geophysics, 81(5).

Luo, Y. and Schuster, G. [1991] Wave-equation traveltime inversion. Geophysics, 56, 645-653.

Shen, P. and Symes, W. [2008] Automatic velocity analysis via shot profile migration. Geophysics, 73(5), VE49-VE59.

Tarantola, A. [1984] Inversion of seismic reflection data in the acoustic approximation. Geophysics, 49(8), 12591266.

Van Leeuwen, T. and Hermann, F. [2013] Mitigating local minima in full-waveform inversion by expanding the search space. Geophysical Journal International, 195(1).

Vigh, D., Jiao, K., Cheng, X., Sun, D. and Lewis, W. [2016] Earth-model building from shallow to deep with fullwaveform inversion. The Leading Edge, 35, 1025-1030.

Virieux, J. and Operto, S. [2009] An overview of fullwaveform inversion in exploration geophysics. Geophysics, 74(6), 127-152.

Wang, C., Yingst, D., Farmer, P. and Leveille, J. [2016] Full-waveform inversion with the reconstructed wavefield method. Expanded Abstracts, Society of Exploration Geophysicists, Dallas, TX, 1237.

Warner, M. and Guasch, L. [2016] Adaptive waveform inversion: Theory. Geophysics, 81(6), R429.

Xu, S., Wang, D., Chen, F., Zhang, Y. and Lambare, G. [2012] Full Waveform Inversion for Reflected Seismic Data. Expanded Abstracts, EAGE, Copenhagen. 